\title{
Older adults have an altered chylomicron response to a high-fat meal
}

\author{
Amber M. Milan ${ }^{1}$, Anu Nuora ${ }^{2}$, Shikha Pundir ${ }^{1}$, Chantal A. Pileggi ${ }^{1}$, James F. Markworth ${ }^{1}$, \\ Kaisa M. Linderborg ${ }^{2}$ and David Cameron-Smith ${ }^{1 *}$ \\ ${ }^{1}$ Liggins Institute, University of Auckland, 85 Park Road, Grafton, Private Bag 92019, Auckland 1023, New Zealand \\ ${ }^{2}$ Food Chemistry and Food Development, Department of Biochemistry, University of Turku, Vatselankatu 2, \\ FI-20014 Turku, Finland
}

(Submitted 9 July 2015 - Final revision received 4 November 2015 - Accepted 16 November 2015 - First published online 15 January 2016)

\section{Abstract}

Ageing is associated with a prolonged and exaggerated postprandial lipaemia. This study aimed to examine the contribution of alterations in chylomicron synthesis, size and lipid composition to increased lipaemia. Healthy older (60-75 years; $n$ 15) and younger (20-25 years; $n$ 15) subjects consumed a high-fat breakfast. Chylomicron dynamics and fatty acid composition were analysed for $5 \mathrm{~h}$ in the postprandial state. Plasma TAG levels were elevated following the meal in the older subjects, relative to younger subjects $(P<0 \cdot 01)$. For older subjects compared with younger subjects, circulating chylomicron particle size was smaller $(P<0.05)$, with greater apoB content $(P<0.05)$ at all postprandial time points. However, total chylomicron TAG concentration between the groups was unaltered post-meal. Compared with younger subjects, the older subjects exhibited a greater proportion of oleic acid in the TAG and phospholipid (PL) fraction $(P<0 \cdot 05)$, plus lower proportions of linoleic acid in the TAG fraction of the chylomicrons $(P<0 \cdot 01)$. Thus, following the ingestion of a high-fat meal, older individuals demonstrate both smaller, more numerous chylomicrons, with a greater total MUFA and lower PUFA contents. These data suggest that the increased postprandial lipaemia of ageing cannot be attributed to increased chylomicron TAG. Rather, ageing is associated with changes in chylomicron particle size, apoB content and fatty acid composition of the chylomicron TAG and PL fractions.

Key words: Cholesterol: Diet and dietary lipids: Lipidomics: Lipoproteins: Nutrition: Phospholipids: TAG

Postprandial lipaemia, characterised by increased concentrations of TAG-rich chylomicron and remnant particles, exerts a significant and often unappreciated impact on CVD $\operatorname{risk}^{(1)}$. Chylomicrons are synthesised within the small intestinal enterocyte, with a lipid composition highly reflective of the previous meal ingestion ${ }^{(2,3)}$. Chylomicrons tend to increase in size with increasing quantities of ingested lipids, but are also larger when composed of proportionally more longer-chain and less saturated $\mathrm{TAG}^{(4,5)}$. Although larger chylomicrons are cleared more slowly than smaller particles, the total number of particles competing for removal has been shown to be the rate-limiting factor in postprandial chylomicron and remnant clearance ${ }^{(6)}$, with chylomicrons being the preferential lipolytic substrate over remnants $^{(7,8)}$.

Older individuals experience greater postprandial lipaemia ${ }^{(9-15)}$. The current evidence suggests that ageing is associated with an increased TAG content of chylomicrons ${ }^{(14,15)}$. Further, measurements of postprandial chylomicron dynamics in older adults have been inconsistent and are frequently reliant on proxy measures such as fat-soluble vitamin appearance ${ }^{(12-15)}$ or apoB-48:TAG ratios $^{(16)}$. Direct measurement of chylomicron particle size with ageing is lacking and this can be quantified by dynamic light scattering methods ${ }^{(17)}$. As chylomicron size dictates lipolytic clearance, age-related differences in chylomicron composition and size could contribute to the reported impaired chylomicron remnant clearance and hypertriacylglycerolaemia in elderly adults ${ }^{(15)}$.

Fat meal loads used in previous studies to induce postprandial lipaemia have varied widely and have often been limited to simple fat boluses that are poorly representative of a typical meal ${ }^{(18)}$. As macronutrients exert differential metabolic responses when ingested alone ${ }^{(19)}$ or in unison ${ }^{(19,20)}$, it is of considerable interest to understand the metabolic response to representative mixed meals. High-fat and high-carbohydrate meals representative of a typical Western diet have increasingly been used to study postprandial lipaemia in healthy ${ }^{(21-23)}$ and metabolically compromised populations ${ }^{(24,25)}$. Thus, in this study, direct measurement of the effect of age on chylomicron dynamics and composition following a high-fat (and highcarbohydrate) meal, representative of a Western diet, was conducted. Analysis was performed to examine chylomicron particle size, apoB concentration and fatty acid composition of both the chylomicron TAG and the phospholipid (PL) fractions in a healthy younger and older adult population. We hypothesised that an exaggerated postprandial lipaemic

Abbreviations: CMRF, chylomicron-rich fraction; PL, phospholipid; TRL, TAG-rich lipoproteins.

* Corresponding author: Professor D. Cameron-Smith, fax +64 09373 7039, email d.cameron-smith@auckland.ac.nz 
response in older adults would be due to the formation of larger chylomicrons containing greater TAG that would delay clearance. We further aimed to describe the fatty acid composition of both the chylomicron TAG and the PL fraction in older adults.

\section{Methods}

\section{Subject selection}

In total, thirty healthy, community-dwelling subjects ( $n 7$ young females, $n 8$ young males, $n 9$ older females, $n 6$ older males) from the Auckland region were recruited through newspaper advertisements and from the university community to participate in the study. Eligible subjects were required to have a BMI between 18 and $30 \mathrm{~kg} / \mathrm{m}^{2}$ and be between the ages of 20-25 and 60-75 years. Individuals with a history of CVD or metabolic disease/conditions including diabetes or thyroid conditions or who used medications that may interfere with study end points (i.e. anti-inflammatory drugs, statin drugs) were not eligible for participation. All subjects reported that they were non-smokers. This study was conducted according to the guidelines laid down in the Declaration of Helsinki, and all procedures involving human subjects were approved by the University of Auckland Human Participants and Ethics Committee (ref no. 8026). Written informed consent was obtained from all subjects. This study was registered prospectively at Australian New Zealand Clinical Trials Registry at anzctr.org.au (ID: ACTRN12612000515897).

\section{Study procedures}

The present study was a cross-sectional, postprandial trial using a high-fat breakfast, chosen as a standard test meal with high fat $(62.2 \mathrm{~g})$ and protein content, also representative of a high-fat restaurant breakfast meal ${ }^{(23)}$ (Table 1 ). Subjects were asked to abstain from vigorous physical activity, high-fat foods,

Table 1. Macronutrient and fatty acid composition of the breakfast meal (Mean values with their standard errors)

\begin{tabular}{|c|c|c|}
\hline Meal components & Value & SEM \\
\hline \multicolumn{3}{|l|}{ Macronutrients ${ }^{*}$} \\
\hline Fat $(\mathrm{g})$ & $62 \cdot 2$ & - \\
\hline Carbohydrates (g) & 77.4 & - \\
\hline Protein $(\mathrm{g})$ & 49.8 & - \\
\hline Energy (kJ) & 4530 & - \\
\hline \multicolumn{3}{|c|}{ Fatty acid mass proportion $(\mathrm{g} / 100 \mathrm{~g}) \dagger$} \\
\hline $14: 0$ & $2 \cdot 9$ & 0.13 \\
\hline $16: 0$ & $18 \cdot 1$ & 0.60 \\
\hline $16: 1 n-7$ & 1.8 & 0.10 \\
\hline $18: 0$ & 9.6 & 0.40 \\
\hline $18: 1 n-7$ & 1.7 & 0.07 \\
\hline $18: 1 n-9$ & $50 \cdot 0$ & 1.70 \\
\hline $18: 1 n-9$ trans & 1.3 & 0.90 \\
\hline $18: 2 n-6$ & $8 \cdot 7$ & 0.30 \\
\hline $18: 3 n-3$ & 2.5 & 0.10 \\
\hline Others $\ddagger$ & 3.35 & 0.50 \\
\hline \multicolumn{3}{|c|}{$\begin{array}{l}\text { Meal consisting of two sausage egg muffin sandwiches containing English muffin } \\
\text { (wheat), egg, sausage patty (beef), cheese slice and rapeseed oil, with two hash } \\
\text { browns containing potatoes and rapeseed oil. Values presented are based on } \\
\text { nutrient panel data obtained from the website of the fast food restaurant. } \\
+n \text {. }\end{array}$} \\
\hline
\end{tabular}

anti-inflammatory medications and nutritional supplements the day before their visit. Subjects arrived fasted on the morning of their visit; anthropometric data were collected before a catheter was inserted into an antecubital vein and a baseline sample (time $0 \mathrm{~h}$ ) was obtained followed by consumption of the test breakfast. Subject physical activity was limited to sedentary activities within the clinical room of the study facility. Blood samples were collected after the meal into serum- and EDTA-containing blood collection tubes (Becton Dickinson) every hour for $5 \mathrm{~h}$. Serum tubes were left to clot at room temperature for $15 \mathrm{~min}$ before all the tubes were centrifuged at $1500 \boldsymbol{g}$ for $15 \mathrm{~min}$ at $4^{\circ} \mathrm{C}$ for serum and plasma separation. An aliquot of plasma was maintained at $4^{\circ} \mathrm{C}$ for chylomicron-rich fraction (CMRF) separation within $6 \mathrm{~h}$, and the remaining plasma was collected in pyrogen-free microtubes and stored at $-20^{\circ} \mathrm{C}$ until analysis.

\section{Chylomicron-rich fraction isolation}

The CMRF, containing chylomicrons and their large remnants, was separated in 4.7-ml OptiSeal tubes (Beckman Coulter) in an Optima MAX-XP ultracentrifuge using a TLA-110 rotor (Beckman Coulter), adapted from Oikawa et al. ${ }^{(26)}$. Densitygradient saline solutions were prepared with $\mathrm{NaCl}$ and $0.005 \%$ EDTA (Sigma-Aldrich) according to Naito ${ }^{(27)}$, and separation protocols were based on the studies of Kupke \& WörzZeugner $^{(28)}$. In brief, $3.5 \mathrm{ml}$ plasma was overlaid with $1.2 \mathrm{ml}$ saline solution $(d=1.006 \mathrm{~g} / \mathrm{ml})$ and centrifuged at $117000 \boldsymbol{g}$ for $10 \mathrm{~min}$. The visible top layer was aspirated into microtubes and corrected to a final collection volume of $1.4 \mathrm{ml}$ using saline solution. This provided a standardised dilution factor of the collected CMRF volume relative to initial plasma volume. The $\mathrm{CMRF}$ was stored in pyrogen-free microtubes at $-80^{\circ} \mathrm{C}$ until use.

\section{Chylomicron-rich fraction particle size analysis}

CMRF particle size was measured using a NanoZetasizer S (Malvern Instruments) by dynamic light scattering. If more than one size peak was observed, the mean peak data for the peak within the range of large TAG-rich lipoproteins (TRL) were used $(>70 \mathrm{~nm}$ ) based on the lower range of mean diameters of small chylomicrons $\left(\mathrm{S}_{\mathrm{f}} 400-1000\right)$, as reported by Fraser ${ }^{(29)}$.

\section{Biochemical analysis}

Concentrations of plasma glucose, cholesterol, LDL, HDL, TAG, NEFA and CMRF TAG, apoB, serum alanine transaminase (ALT) and serum aspartate transaminase (AST) were measured using a Hitachi 902 autoanalyser (Hitachi High Technologies Corporation) by enzymatic colorimetric assay (Roche). Plasma insulin concentration was measured using an Abbott AxSYM system (Abbott Laboratories) by microparticle enzyme immunoassay.

\section{Fatty acid composition analysis of meals and chylomicron-rich fraction TAG and phospholipids}

Meals were homogenised using a domestic blender with $600 \mathrm{~g}$ water. Samples were aliquoted into $50-\mathrm{ml}$ centrifuge tubes, 
frozen and stored at $-80^{\circ} \mathrm{C}$ until use. Fatty acid extraction, methylation and GC of meal and CMRF samples were carried out according to protocols described previously ${ }^{(30-32)}$. In short, an internal standard mixture of triheptadecanoin (Sigma-Aldrich) and dinonadecanoylphosphatidylcholine (Sigma-Aldrich) was added to the isolated CMRF. Subsequently, $1.5 \mathrm{ml}$ methanol, $3 \mathrm{ml}$ chloroform and $0.8 \mathrm{ml} 0.88 \% \mathrm{KCl}$ in water were added and the blend was thoroughly vortexed after each addition. Samples were centrifuged at $2000 \boldsymbol{g}$ for $3 \mathrm{~min}$ to separate the layers, and the lower chloroform-rich layer was removed and the upper layer was extracted again ${ }^{(30)}$. Food lipids were extracted just as CMRF after further homogenisation with an Ultra Turrax (IKA). CMRF TAG and PL were isolated from the extracted lipid mixture with solid-phase extraction based on Sep-Pak ${ }^{\circledR}$ Vac 1 cc silica columns (Waters) ${ }^{(31)}$.

Fatty acid methyl esters (FAME) were prepared using the sodium methoxide method. In short, the lipids were suspended into $1 \mathrm{ml}$ dry diethyl ether; subsequently, $25 \mu \mathrm{l}$ methyl acetate and $25 \mu \mathrm{l}$ sodium methoxide were added and the blend was incubated for $5 \mathrm{~min}$ while shaken at times. The reaction was stopped with $6 \mu \mathrm{l}$ acetic acid. Samples were centrifuged at $2000 \boldsymbol{g}$ for $5 \mathrm{~min}$, after which the supernatant was removed and gently evaporated to dryness. The resulting FAME were transferred to $100 \mu \mathrm{l}$ inserts in hexane ${ }^{(32)}$. The FAME were analysed using a gas chromatograph (Shimadzu GC-2010) equipped with an AOC-20i auto injector, a flame ionisation detector (Shimadzu Corporation) and a wall-coated open tubular column DB-23 (60 $\mathrm{m} \times 0.25 \mathrm{~mm}$ i.d., liquid film 0.25 $\mu \mathrm{m}$; Agilent Technologies, J.W. Scientific). Splitless/split injection was used and the split was opened after $1 \mathrm{~min}$. Supelco 37 Component FAME Mix (Supelco), 68D (Nu-Chek-Prep) and GLC-490 (Nu-Chek-Prep) were used as external standards.

\section{Calculations}

The homoeostatic model assessment of insulin resistance (HOMA-IR) was calculated from fasting glucose and insulin concentrations using the equation from the study by Matthews et $a l{ }^{(33)}$. Maximum concentrations $\left(C_{\max }\right)$ were identified as the greatest value in an individual's data set, with maximum peak times $\left(T_{\max }\right)$ identified as the corresponding nominal sampling time. Arithmetic means were used for group $C_{\max }$ comparisons, whereas median values were used for group $T_{\max }$ comparisons. Plasma fatty acids were pooled for mathematical analysis into SFA, MUFA, PUFA and dairy-derived odd-chain fatty acids.

\section{Statistical analyses}

Statistical analyses were carried out using Statistical Package for the Social Sciences version 21 (SPSS; IBM Corporation). Sample size was estimated with a power analysis for a superiority design based on data from a previous study on lipaemia in the elderly $^{(11)}$. To achieve a significance level of $P \leq 0.05$ with $80 \%$ power to detect a between-subject difference of $4.77 \mathrm{mmol} / 1$ per $h$ for TAG incremental AUC (iAUC), a sample size of 30 was necessary. Data are presented as means values with their standard errors except $T_{\max }$, which is presented as the medians and interquartile ranges. iAUC was calculated after subtracting fasting values. Baseline subject characteristics, iAUC and $C_{\max }$ were compared using unpaired Student's $t$ test. $T_{\max }$ was compared using the Mann-Whitney $U$ test using mean ranks. Two-factor repeated-measures ANOVA (time compared withinsubject and age compared between subjects) followed by the Sidak-adjusted post hoc test were used for all multiple comparisons between different groups. Where Mauchly's sphericity test failed, the Huynh-Feldt correction was applied. $\alpha$ was set at $P<0.05$. Heat maps were created using $\mathrm{R}$ software version 2.15.2 using the packages gplots (heatmap.2), RColorBrewer and colorRamps (R Development Core Team).

\section{Results}

\section{Subject characteristics}

A total of thirty subjects completed the study ( $n 15$ younger, $n 15$ older). There were no significant differences between the two age groups for BMI or fasting measurements of plasma glucose, plasma insulin, HOMA-IR, plasma or CMRF TAG or CMRF apoB content or particle size (Table 2). Fasting plasma lipid profiles were within the normal range for both age groups, although total, LDL and HDL-cholesterol levels were all higher in older subjects $(P<0.001, P=0.002$ and $P<0.001$, respectively). Liver function measured by serum ALT and AST were within the normal range for all participants; however, the older participants displayed lower concentrations for each $(P=0.004$ and $P=0 \cdot 001$, respectively).

\section{Plasma glucose, insulin and lipid responses}

Postprandial glucose (Fig. 1(a)) and insulin (Fig. 1(b)) responses to the meal did not differ between younger and older

Table 2. Baseline subject characteristics and fasting plasma, serum and chylomicron-rich fraction lipid profile of older and younger subjects (Mean values with their standard errors)

\begin{tabular}{|c|c|c|c|c|}
\hline \multirow[b]{2}{*}{ Measures } & \multicolumn{2}{|c|}{$\begin{array}{l}\text { Younger subjects } \\
\qquad(n 15)\end{array}$} & \multicolumn{2}{|c|}{$\begin{array}{l}\text { Older subjects } \\
\qquad(n 15) \dagger\end{array}$} \\
\hline & Mean & SEM & Mean & SEM \\
\hline Age (years) & $22 \cdot 7$ & 0.4 & $67 \cdot 3^{\star \star \star}$ & 1.5 \\
\hline $\mathrm{BMI}\left(\mathrm{kg} / \mathrm{m}^{2}\right)$ & $23 \cdot 7$ & 0.8 & 24.4 & 1.0 \\
\hline HOMA-IR & $2 \cdot 0$ & 0.2 & $1 \cdot 7$ & 0.3 \\
\hline \multicolumn{5}{|l|}{ Plasma } \\
\hline Glucose (mmol/l) & $5 \cdot 1$ & 0.1 & $5 \cdot 2$ & $0 \cdot 1$ \\
\hline Insulin $(\mathrm{mmol} / \mathrm{l})$ & $9 \cdot 0$ & 1.0 & $8 \cdot 7$ & 1.9 \\
\hline Total cholesterol $(\mathrm{mmol} / \mathrm{l})$ & $4 \cdot 0$ & 0.2 & $5 \cdot 0^{\star \star \star}$ & 0.2 \\
\hline $\mathrm{LDL}(\mathrm{mmol} / \mathrm{l})$ & $2 \cdot 4$ & 0.2 & $3 \cdot 0^{* *}$ & 0.2 \\
\hline $\mathrm{HDL}(\mathrm{mmol} / \mathrm{l})$ & 1.4 & $0 \cdot 1$ & $1 \cdot 8^{\star \star \star}$ & $0 \cdot 1$ \\
\hline TAG (mmol/l) & 0.82 & 0.07 & 0.91 & 0.07 \\
\hline NEFA (mmol/l) & 0.66 & 0.08 & 0.57 & 0.09 \\
\hline \multicolumn{5}{|l|}{ Serum } \\
\hline ALT (U/I) & $10 \cdot 0$ & $2 \cdot 2$ & $1.9^{* *}$ & 0.8 \\
\hline AST (U/I) & $19 \cdot 1$ & $1 \cdot 7$ & $12 \cdot 1^{\star *}$ & 0.7 \\
\hline \multicolumn{5}{|l|}{ Chylomicron-rich fraction } \\
\hline TAG (mmol/l of plasma) & 0.07 & 0.02 & 0.08 & 0.01 \\
\hline Size $(\mathrm{nm})$ & 191 & 20 & 140 & 24 \\
\hline apoB (mmol/l of plasma) & $2 \cdot 3$ & 0.5 & 3.4 & 0.5 \\
\hline
\end{tabular}

HOMA-IR, homoeostatic model assessment of insulin resistance; ALT, alanine transaminase; AST, aspartate transaminase.

† Significance was determined by unpaired Student's $t$ test: ${ }^{\star \star \star} P<0.001,{ }^{\star \star} P<0.01$ compared with younger subjects. 
(a)

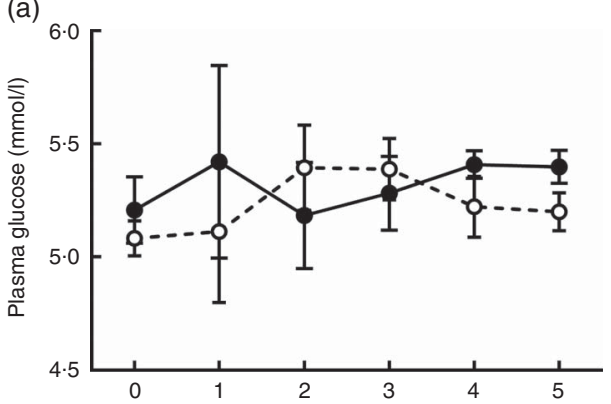

(c)

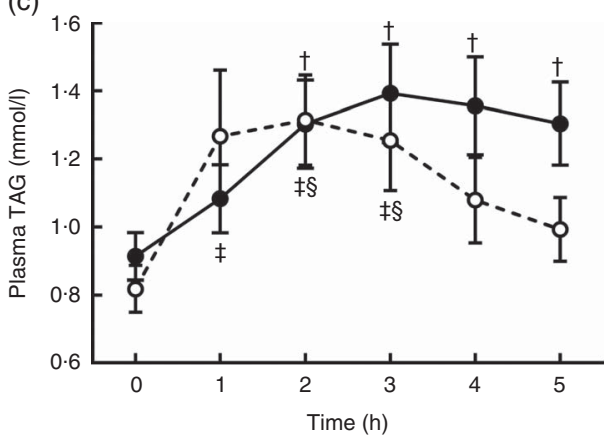

(b)

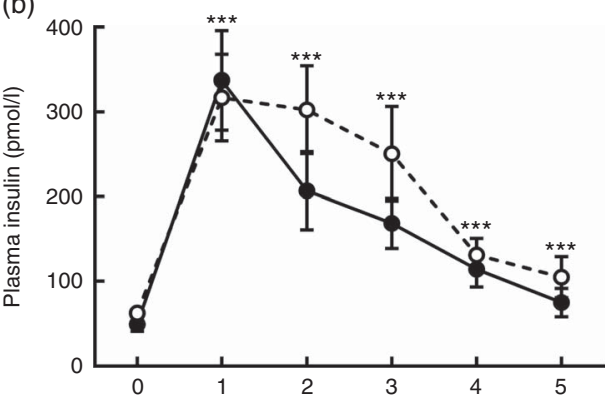

(d)

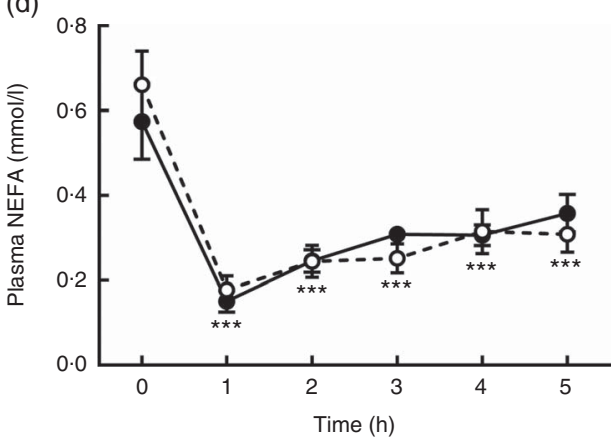

Fig. 1. Postprandial plasma concentrations of glucose (a), insulin (b), TAG (c) and NEFA (d) in older (- - ) and younger (- -O- -) subjects. Values are means ( $n$ 15/ group), with their standard errors. There were no differences in glucose, insulin or NEFA responses between older and younger subjects. There were significant differences over time in the TAG response between older and younger subjects (age $\times$ time interaction of $P<0.01$, two-factor repeated-measures ANOVA). *** $P<0.001$ change from baseline in all subjects; $† P<0.01$, change from baseline in older subjects; $\ddagger P<0.01$ change from baseline in younger subjects; $\S P<0.01$ change from $5 \mathrm{~h}$ in younger subjects (Sidak-corrected post hocs).

subjects. The older group had a delayed median time to achieve peak plasma TAG concentrations relative to the younger group ( $T_{\max } 3$ (IQR 3-4) v. 2 (IQR $1.5-3$ ) h, respectively; $P<0 \cdot 001$ ), reaching peak TAG concentration $\left(C_{\max }\right)$ after $3 \mathrm{~h}(P<0.001 v$. baseline). The younger subjects reached maximum peak concentration at $2 \mathrm{~h}(P=0.02 v$. baseline $)$. The plasma TAG in the younger subjects also returned to baseline by $4 \mathrm{~h}$; however, in the older group, plasma TAG remained significantly elevated at $5 \mathrm{~h}$ post-meal (Fig. 1(c)). As such, we observed a prolonged TAG response in older subjects. This pattern of response did not impact on the iAUC $(0-5 \mathrm{~h})$ response between the younger and the older groups (103.6 (SEM 21.6) $v .102 .5$ (SEM 19.1) $\mathrm{mmol} / \mathrm{l}$, respectively; $P=0.97$; data not shown). Marked suppression of circulating plasma NEFA was observed following the meal, with no difference between younger and older subjects (Fig. 1(d)). Postprandial total plasma cholesterol concentrations decreased in the older but not in the younger participants (Table 3; time-age interaction; $P<0 \cdot 001$ ).

\section{Chylomicron-rich fraction dynamics}

CMRF particle size increased after the meal $(P=0 \cdot 01$; main effect of time, Fig. 2(a)) and was smaller for the older compared with the younger participants $(P=0 \cdot 011$, main effect of age). Owing to variation in time to peak size between individuals, median group maximum peak CMRF particle size and $T_{\max }$ were compared. Overall median peak time was $3 \mathrm{~h}$ post-meal
Table 3. Postprandial total cholesterol in older and younger subjects (Mean values with their standard errors)

\begin{tabular}{lcclll}
\hline & \multicolumn{2}{c}{ Younger subjects $(n$ 15) } & & \multicolumn{2}{c}{ Older subjects $(n$ 15) $\dagger$} \\
\cline { 2 - 3 } \cline { 6 - 6 } mmol/l & Mean & SEM & & Mean & SEM \\
\hline Baseline & 3.99 & 0.18 & & 5.04 & 0.22 \\
$1 \mathrm{~h}$ & 3.93 & 0.17 & & 4.92 & 0.16 \\
$2 \mathrm{~h}$ & 3.95 & 0.18 & & $4.84^{\star \star}$ & 0.18 \\
$3 \mathrm{~h}$ & 3.86 & 0.18 & & $4.75^{\star \star}$ & 0.16 \\
$4 \mathrm{~h}$ & 3.93 & 0.20 & & $4.75^{\star \star \star} \S$ & 0.16 \\
$5 \mathrm{~h}$ & 3.97 & 0.19 & & $4.80^{\star \star}$ & 0.16 \\
\hline
\end{tabular}

† Significance was determined by two-factor repeated-measure ANOVA with Sidakcorrected post hoc analysis: ${ }^{\star \star} P<0.01$, ${ }^{\star \star \star} P<0.001$ compared with baseline within age group; $\S P<0.01$ compared with $1 \mathrm{~h}$. All time point comparisons between younger and older subjects were significant to $P<0.01$.

$\left(T_{\max } 3(\mathrm{IQR} 2-3 \cdot 5) \quad v .2\right.$ (IQR $\left.1-4\right) \mathrm{h}$, younger $v$. older, respectively; $P=0 \cdot 467)$. Maximum CMRF particle size tended to be smaller in the older subjects ( $C_{\max } 321$ (SEM 29) and 390 (SEM 22) nm, older $v$. younger, respectively; $P=0 \cdot 072$ ). As CMRF particle size was not different at baseline between age groups, post hoc analysis was conducted to determine age-group postprandial changes in size, despite no time-age interaction. The older group's CMRF particle size remained constant after the meal, whereas the younger group's CMRF particle size increased, resulting in an age difference in size at $2-3 \mathrm{~h}$ $(P=0.022$ and $P=0.007$, respectively). Postprandial CMRF TAG 

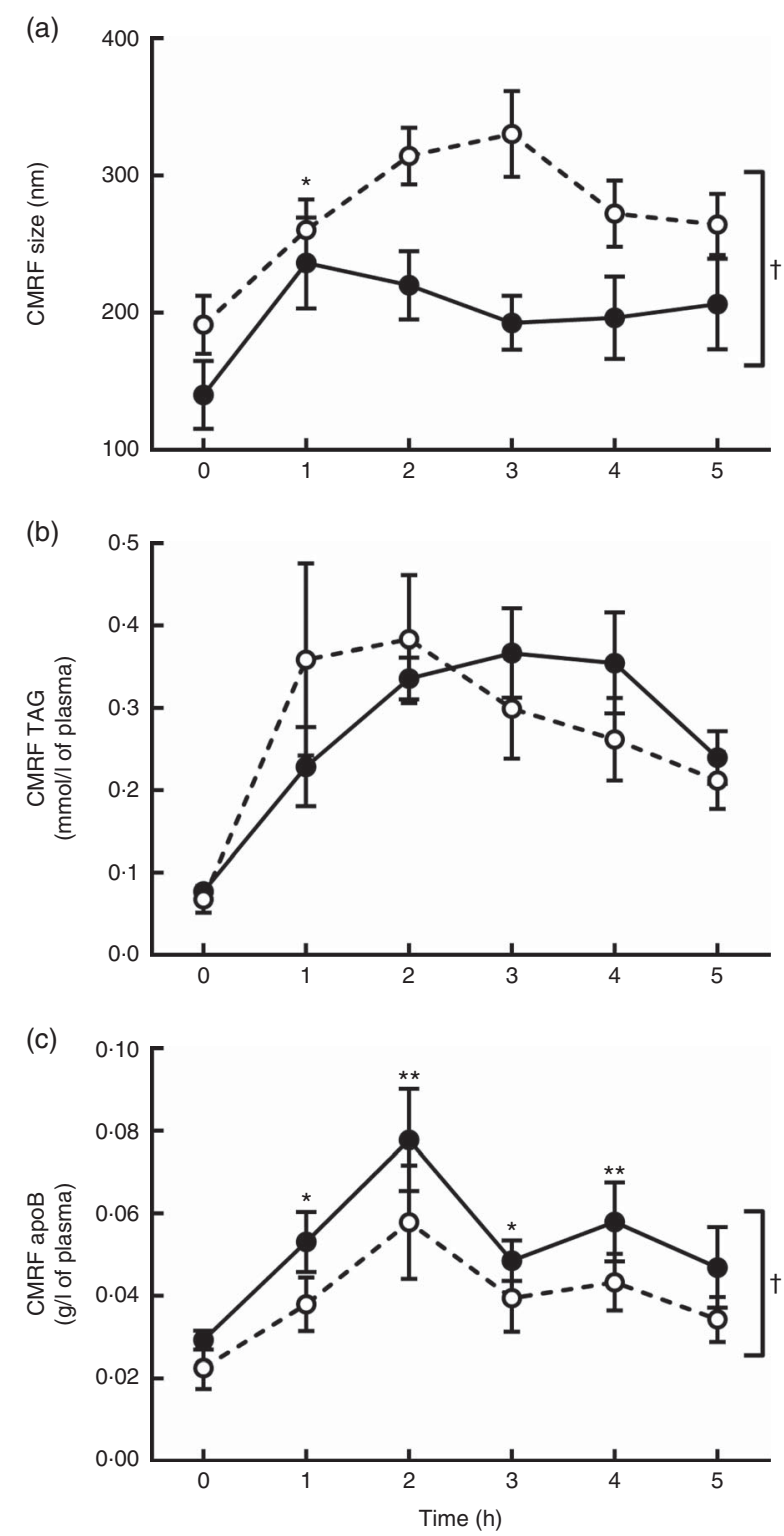

Fig. 2. Postprandial chylomicron-rich fraction (CMRF) particle size (a), TAG concentration (b) and apoB concentration (c) in older (- - ) and younger

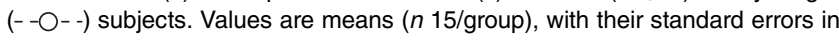
$\mathrm{nm}$ for particle size, $\mathrm{mmol} / \mathrm{l}$ of plasma for TAG and $\mathrm{g} / \mathrm{l}$ of plasma for apoB. There were no differences in CMRF TAG response concentrations between older and younger subjects. There were significant main age differences in the particle size and apoB responses between older and younger subjects (age effect marked ( $\dagger$ ) of $P<0.05$ each, two-factor repeated-measures ANOVA). ${ }^{*} P<0.05$ change from baseline in all subjects; ${ }^{\star *} P<0.01$ change from baseline in all subjects (Sidak-corrected post hocs).

concentration was not different between older and younger subjects $(P=0 \cdot 894$; Fig. 2(b)).

Changes in CMRF particle numbers were assessed by apoB concentrations. Older subjects had higher apoB concentrations than younger subjects (mean postprandial concentration $0-5 \mathrm{~h}$ of $5.3($ SEM 0.4$) v .4 .2($ SEM 0.4$) \times 10^{-2} \mathrm{~g} / 1$, respectively; $P=0.035$; Fig. 2(c)). Overall, apoB concentrations peaked at $2 \mathrm{~h}$ post-meal $(P=0.001)$ returning to comparable baseline concentrations by $5 \mathrm{~h}$.

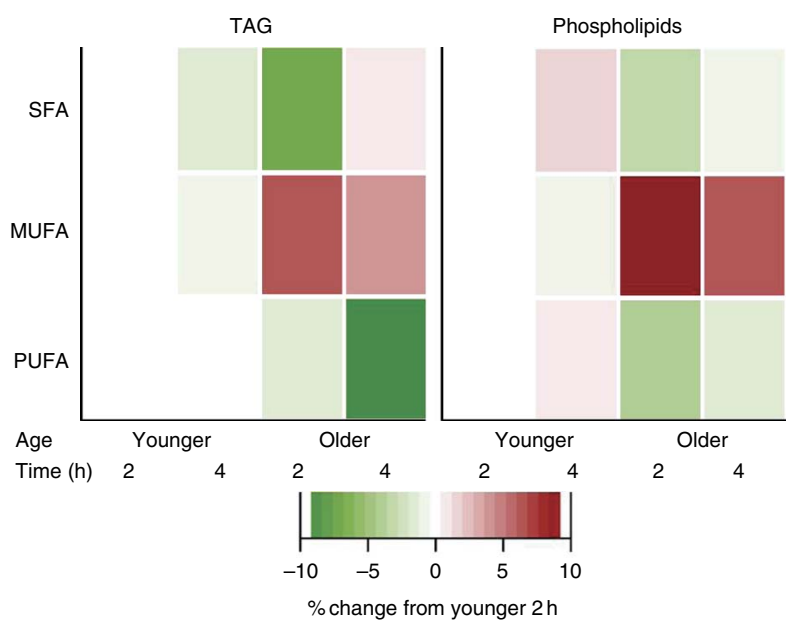

Fig. 3. Heat map of distribution changes of chylomicron-rich fraction SFA, MUFA and PUFA after the meal of older and younger subjects. Values are presented as mean per cent changes relative to younger-subject 2-h mass proportion for each saturation class. $\square, 0 \%$ change from younger $2 \mathrm{~h}$; , negative percentage changes; $\square$, positive percentage changes.

\section{Chylomicron-rich fraction TAG and phospholipid fatty acid composition}

Comparison of chylomicron-rich fraction TAG composition between ages. Older subjects had greater proportions of MUFA in their CMRF TAG $(5.6 \%$ greater proportion in older $v$. younger subjects; $P=0.014$; Fig. 3 ). In addition, $4 \mathrm{~h}$ after the meal, older subjects had a lower proportion of PUFA in their CMRF TAG (9.4\% lower proportion in older $v$. younger subjects; $P=0.004$ ). Older participants' CMRF TAG tended to have lower proportions of SFA at $2 \mathrm{~h}(7 \cdot 0 \%$ lower proportion in older $v$. younger subjects; $P=0.079$ ), a trend that was lost by $4 \mathrm{~h}$.

These differences in MUFA and PUFA CMRF TAG composition were attributable to differences in the major fatty acids oleic acid and linoleic acid. The older group's CMRF TAG had relatively more oleic acid (18:1n-9; $P=0.033$; Fig. 4(a)). Although $18: 2 n-6$ (linoleic acid) was found in equal proportions in both groups' CMRF TAG at $2 \mathrm{~h}$, by $4 \mathrm{~h}$ the older subjects had lower proportions $(P=0 \cdot 003)$. The older group had lower proportions of $18: 0$ (stearic acid) at $2 \mathrm{~h}$ compared with younger participants $(P=0 \cdot 009)$, whereas these age differences were not apparent after $4 \mathrm{~h}$. Older subjects had lower proportions of $15: 1$ at $2 \mathrm{~h}$ compared with the younger group $(P=0 \cdot 037)$.

Pooling of the dairy-derived odd-chain fatty acids (15:0, $15: 1,17: 0,17: 1)$ showed higher proportions in the CMRF TAG of older subjects $(P<0 \cdot 001)$. In both groups, CMRF TAG composition changed over the postprandial period. From 2 to $4 \mathrm{~h}$ after the meal, proportions of CMRF TAG 20:4n-6 (arachidonic acid) and $22: 6 n-3$ (DHA) increased $(P=0.002$ and $P=0 \cdot 042$, respectively).

Comparison of chylomicron-rich fraction phospholipid composition between ages. In both older and younger subjects, CMRF PL composition changed over the postprandial period. At $4 \mathrm{~h}, \mathrm{CMRF} \mathrm{PL}$ contained relatively more PUFA $(P=0.034)$, less SFA $(P=0.042)$ and tended to contain more MUFA $(P=0.052)$ than at $2 \mathrm{~h}$ (Fig. 3). This difference in the later 
(a)

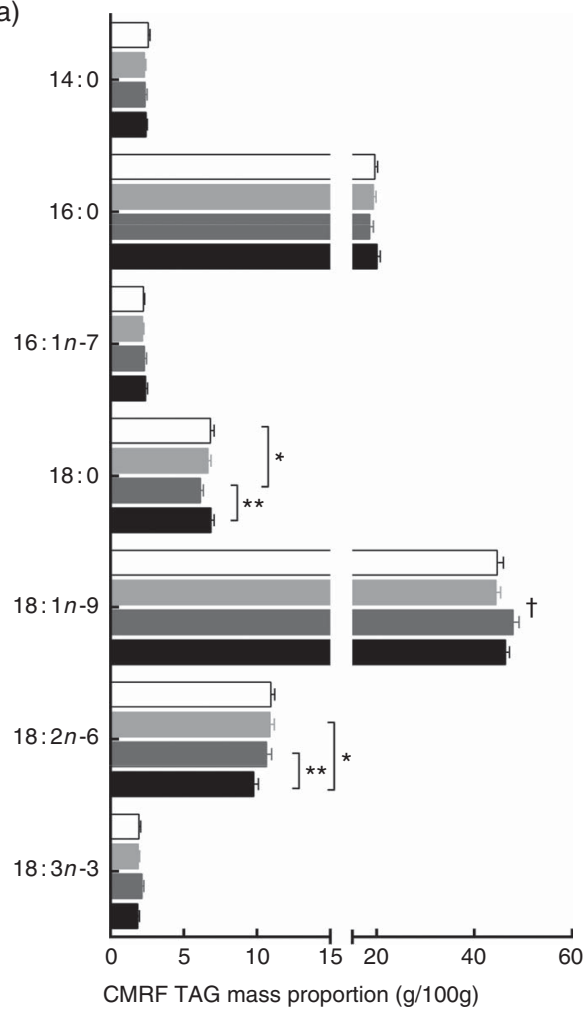

(b)

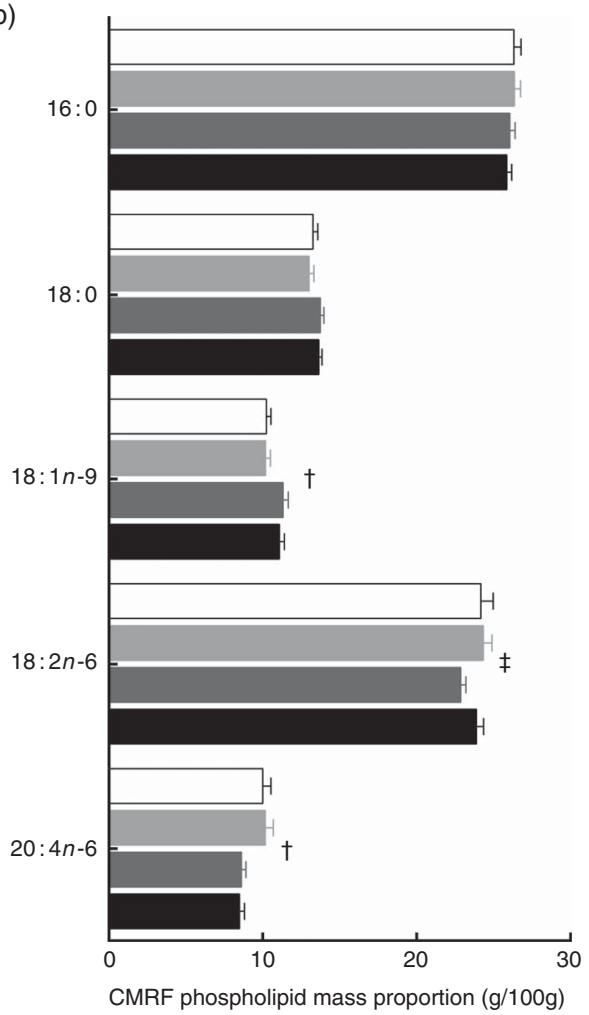

Fig. 4. Major chylomicron-rich fraction (CMRF) TAG (a) and phospholipid (b) fatty acid composition of younger subjects at $2 \mathrm{~h}$ ( $\square$ ) and $4 \mathrm{~h}$ ( $\square$ ) and older subjects at $2 \mathrm{~h}$ ) and $4 \mathrm{~h}(\square)$ as mass proportions $(\mathrm{g} / 100 \mathrm{~g})$. Data marked $(\dagger)$ differ with age, and data marked $(\ddagger)$ differ with time; $P<0.05$ for age and time by two-factor repeatedmeasures ANOVA. Age $\times$ time interaction post hocs (Sidak-corrected within each acid) are indicated as ${ }^{*} P<0.05$, ${ }^{* *} P<0.01$ between younger and older subjects at specified time points.

postprandial period was characterised by relatively more linoleic acid (18:2n-6;P=0.023) than at $2 \mathrm{~h}$ (Fig. 4(b)).

The CMRF PL of the older subjects contained relatively more MUFA ( $8.7 \%$ greater proportion in older $v$. younger subjects; $P=0.038)$, less PUFA $(3.3 \%$ lower proportion in older $v$. younger subjects; $P=0.032$ ) and tended to contain less SFA ( $3.2 \%$ lower proportion in older $v$. younger subjects; $P=0.085$; Fig. 3) relative to younger subjects. Arachidonic acid was found in lower concentrations in older subjects $(P=0 \cdot 018)$.

Similar to the CMRF TAG, the CMRF PL in the older group contained greater proportions of oleic acid (18:1n-9; $P=0.028)$. Elaidic acid (18: $1 n$-9trans at $2 \mathrm{~h} ; P=0.001)$ and 18:2n-6trans $(P=0.012)$ were also more abundant in the CMRF PL of older participants; this was accompanied by relatively higher levels of the dairy-derived odd-chain fatty acids $(P=0.001)$, particularly $15: 0$ and $15: 1 \quad(P=0.018$ and $P=0.003$, respectively). Furthermore, the older subjects had greater proportions of the PUFA docosapentaenoic acid (22:5n-3; $P=0.013)$ and tended to have relatively more EPA $(P=0 \cdot 058)$.

\section{Discussion}

Following a high-fat meal, the older participants of the present study had smaller chylomicron particles with a greater proportion of oleic acid and a lower PUFA content over a 5-h postprandial period. This study highlights that there are subtle changes in the dynamics governing lipid absorption, which occur along with differences in relative absorption and/or clearance of specific fatty acids, predominately oleic acid. Furthermore, our data do not support either altered appearance or delayed chylomicron clearance as a major regulator of the exaggerated lipaemia, typically present in older individuals. Rather, this study shows that the major contributant to the heightened postprandial TAG response is in the nonchylomicron fraction and includes other TAG-rich particles including VLDL and chylomicron remnants ${ }^{(11)}$.

Consistent with previous reports, our data demonstrate prolonged postprandial triacylglycerolaemia in older adults ${ }^{(9-15)}$. However, within this literature, a few studies have addressed the dynamics and role of chylomicron structure and composition in this hypertriacylglycerolaemic response. In the present study, the concentration of CMRF TAG was not different between older and younger participants. Previous studies reporting differences in chylomicron $\mathrm{TAG}^{(13-15)}$ have shown greater TAG content in large, rather than small, TRL in the elderly $^{(12)}$, plus a greater postprandial NEFA spillover from exogenous TRL ${ }^{(11)}$. Neither mechanism was demonstrated in the present study. Rather, our data implicate alterations of small TRL (endogenous VLDL and small chylomicron remnants) production and clearance as a major determinant of the sustained postprandial hypertriacylglycerolaemia in the elderly. It must be noted that there were no age differences in BMI, HOMA-IR, fasting TAG and NEFA or indications of hepatic 
dysfunction in this present study. Previously, these factors have been shown to adversely affect postprandial chylomicron dynamics $^{(11)}$. Furthermore, our findings are presented in the context of a mixed breakfast meal, rather than in response to the ingestion of isolated lipid loads.

In this study, the older participants had a maximum chylomicron particle size, on average $18 \%$ smaller and $27 \%$ more numerous compared with the younger group. Importantly, chylomicron sizes were measured directly through dynamic light scattering, a technique used previously for chylomicron and lipoprotein size analyses ${ }^{(17,34)}$, although rarely in the context of postprandial dynamics ${ }^{(35)}$. These smaller and more numerous TRL are a reported feature of insulin resistance $^{(36)}$ and certain types of dyslipidaemia ${ }^{(37)}$. Smaller and more abundant TRL may be due to intestinal over-production of apoB- $48^{(38,39)}$ and/or failed suppression of postprandial large VLDL production ${ }^{(40)}$. However, in this study, no age difference in fasting insulin sensitivity, as measured by HOMA-IR, were observed. Thus, the mechanisms contributing to the increased concentrations of smaller and more numerous chylomicron particles were not determined in the present study.

Differences in chylomicron particle size and number may have important implications for chylomicron clearance and potentially for atherosclerotic risk in these older subjects. Smaller chylomicron particle size has been shown to retard clearance rate $^{(41)}$; however, more recent investigations have shown that this may be an artifact of unmatched number of particles ${ }^{(6)}$, indicating that larger numbers, often corresponding to smaller size, are cleared more slowly. Smaller and greater circulating numbers of chylomicron remnants, due to their ability to penetrate the arterial intima, have been shown to increase atherosclerotic risk $^{(1)}$. However, our results suggest that the large CMRF, although containing relatively smaller particles, is cleared efficiently, whereas the small TRL fraction may persist.

In addition to the alterations in the size and number of circulating chylomicrons, differences in the lipid composition of both the TAG and the PL fractions were measured. The older individuals had greater proportions of MUFA, particularly oleic acid, in chylomicron TAG, with a corresponding lowering of the proportion of PUFA. These compositional differences were also present in the PL fraction. Our findings correspond with reports of age-related differences in adipose and circulating TAG composition with age-related declines in $\mathrm{SFA}^{(42)}$, linoleic acid $(18: 2 n-6)^{(43)}$ and total PUFA contents ${ }^{(44)}$, along with increases in MUFA content ${ }^{(42)}$. Similarly, our findings of lower chylomicron PL PUFA are similar to what has been previously demonstrated in elderly populations ${ }^{(44-46)}$. Habitual diet or health status has been suggested to impact these age-related compositional differences ${ }^{(43,45)}$. Indeed, the post-meal plasma fatty acid composition has previously been shown to correspond poorly with meal fatty acid composition, particularly regarding plasma MUFA content in older subjects, suggesting an important impact of habitual diet ${ }^{(47)}$. In contrast, meal composition has been reported to significantly impact postprandial TAG in young subjects ${ }^{(48)}$, supporting a difference in post-meal fatty acid metabolism between old and young individuals. Furthermore, as our postprandial findings match longer-term evaluations of circulating fatty acids in elderly populations, the metabolic handling of fatty acids may contribute to age-related fatty acid differences in the elderly.

There are likely to be several mechanisms exerting influence on the differences in chylomicron fatty acid composition between the younger and the older subjects following the consumption of an identical meal, including possible differences in fatty acid absorption and clearance from TRL. Enterocyte fatty acid metabolism may be involved as chylomicron incorporation of fat-soluble vitamins has suggested age-related differences in postprandial absorption ${ }^{(12,14)}$. The greater proportions of MUFA observed in the older group may indicate enhanced intestinal absorption of oleic acid. This is supported by animal models indicating that uptake of oleic acid ${ }^{(49)}$, linoleic acid ${ }^{(50)}$ and SFA ${ }^{(51)}$ are increased in aged rats, fitting with our data. In addition, chylomicron TAG are known to contain proportions of fatty acids originating from enterocyte storage pools, containing fatty acids from the previous meal ${ }^{(52,53)}$ as well as early postprandial contributions from NEFA ${ }^{(52)}$. It has been suggested that in insulin-resistant states the intestines may store greater quantities of fat, contributing to aberrant apoB production $^{(54)}$. It is unknown whether differences in intestinal enterocyte storage pools between age groups may have contributed to postprandial fatty acid compositional differences. However, it is probable that the predominant mediator is exchange with either circulating free or lipoprotein-derived PL and TAG or the presence of endogenous large TRL. Indeed, the postprandial reductions in total cholesterol we observed in the older group suggest increased inter-lipoprotein transfer; however, measures of this activity such as cholesterol ester transfer activity were not assessed. Furthermore, the origins of altered proportions of fatty acids in any endogenous lipoproteins, such as greater MUFA content, remain unknown.

The postprandial age-related differences in older participants' chylomicron composition may have metabolic implications outside of transient effects on postprandial lipaemia. As older people incorporate ingested TAG at a greater rate into small TRL rather than chylomicrons ${ }^{(11)}$, any differences in fatty acid uptake will be amplified with the increased residence time of these remnants ${ }^{(9,15)}$. In addition, the increased surface area: volume ratio of chylomicrons in the older group implies proportionally more PL, magnifying the impact of these compositional differences. Specifically, enhanced uptake of oleic acid could have implications for uptake of other fatty acids, as long-chain fatty acid uptake into the enterocyte is competitive $^{(55)}$, and could explain the lower proportions of PUFA found in older subjects' chylomicron TAG. Furthermore, the fatty acid composition differences reported in these older adults have been identified as features in individuals with metabolic dysfunction; lower serum linoleic acid has been associated with an increased risk of developing impaired fasting glucose or type 2 diabetes mellitus in middle-aged men ${ }^{(56)}$. In addition, as we show that some age-related compositional differences are dependent on postprandial sampling time, differences in rates of uptake or clearance may contribute to these age-related differences, highlighting a downfall of compositional profile analysis from a single time point when temporal metabolic differences exist between populations. 


\section{Conclusions}

The exaggerated postprandial lipaemia typical of older individuals is not characterised by elevated chylomicron TAG. This study demonstrated that in an older healthy cohort there is no impairment of large chylomicron clearance. Despite this preservation of a chylomicron response to a high-fat meal, the resultant chylomicrons are smaller and more numerous in the elderly, containing greater proportions of MUFA and lower proportions of PUFA. The implications of these fundamental differences in chylomicron dynamics and composition are unknown in the context of CVD risk and warrant further investigation.

\section{Acknowledgements}

The authors thank the study participants, the support of the Maurice \& Agnes Paykel Clinical Research Unit, particularly Janene Biggs, Ben Albert, Christine Brennan, Vic Shao-Chih Chiang and Wonjoo Lee and the lipid analysis assistance of Karlo Villa.

This study was funded by the Liggins Institute Trust through project no. 3701462. The Liggins Institute Trust had no role in the design, analysis or writing of this article.

A. M. M. carried out laboratory analysis and data interpretation and drafted the manuscript. A. N. carried out lipidomic analysis. A. M. M., S. P. and C. A. P. were involved in the coordination, management and implementation of the clinical trial. J. F. M. provided laboratory supervision. J. F. M. and K. M. L. critically evaluated the manuscript. K. M. L. supervised lipidomic analysis. D. C.-S. formulated the research question, initiated and supervised all aspects of the study. All the authors approved the final version of the manuscript for submission.

The authors declare that there are no conflicts of interest.

\section{References}

1. Nordestgaard BG \& Freiberg JJ (2011) Clinical relevance of non-fasting and postprandial hypertriglyceridemia and remnant cholesterol. Curr Vasc Pharmacol 9, 281-286.

2. Bysted A, Hølmer G, Lund P, et al. (2005) Effect of dietary fatty acids on the postprandial fatty acid composition of triacylglycerol-rich lipoproteins in healthy male subjects. Eur J Clin Nutr 59, 24-34.

3. Summers LKM, Barnes SC, Fielding BA, et al. (2000) Uptake of individual fatty acids into adipose tissue in relation to their presence in the diet. Am J Clin Nutr 71, 1470-1477.

4. Perez-Martinez P, Ordovas JM, Garcia-Rios A, et al. (2011) Consumption of diets with different type of fat influences triacylglycerols-rich lipoproteins particle number and size during the postprandial state. Nutr Metab Cardiovasc Dis 21, $39-45$.

5. Jackson KG, Zampelas A, Knapper JME, et al. (2000) Differences in glucose-dependent insulinotrophic polypeptide hormone and hepatic lipase in subjects of southern and northern Europe: implications for postprandial lipemia. Am J Clin Nutr 71, 13-20.

6. Martins IJ, Mortimer B-C, Miller J, et al. (1996) Effects of particle size and number on the plasma clearance of chylomicrons and remnants. J Lipid Res 37, 2696-2705.
7. Bjorkegren J, Packard CJ, Hamsten A, et al. (1996) Accumulation of large very low density lipoprotein in plasma during intravenous infusion of a chylomicron-like triglyceride emulsion reflects competition for a common lipolytic pathway. J Lipid Res 37, 76-86.

8. Karpe F, Olivecrona T, Hamsten A, et al. (1997) Chylomicron/ chylomicron remnant turnover in humans: evidence for margination of chylomicrons and poor conversion of larger to smaller chylomicron remnants. J Lipid Res 38, 949-961.

9. Cohn JS, McNamara JR, Cohn SD, et al. (1988) Postprandial plasma lipoprotein changes in human subjects of different ages. J Lipid Res 29, 469-479.

10. Puga GM, Meyer C, Mandarino LJ, et al. (2013) Increased plasma availability of L-arginine in the postprandial period decreases the postprandial lipemia in older adults. Nutrition 29, 81-88.

11. Puga GM, Meyer C, Everman S, et al. (2011) Postprandial lipemia in the elderly involves increased incorporation of ingested fat in plasma free fatty acids and small (Sf 20-400) triglyceride-rich lipoproteins. Am J Physiol Endocrinol Metab 301, E356-E361.

12. Borel P, Mekki N, Boirie Y, et al. (1997) Postprandial chylomicron and plasma vitamin $\mathrm{E}$ responses in healthy older subjects compared with younger ones. Eur J Clin Invest 27, 812-821.

13. Borel P, Mekki N, Boirie Y, et al. (1998) Comparison of the postprandial plasma vitamin A response in young and older adults. J Gerontol A Biol Sci Med Sci 53, B133-B140.

14. Cardinault N, Tyssandier V, Grolier P, et al. (2003) Comparison of the postprandial chylomicron carotenoid responses in young and older subjects. Eur J Nutr 42, 315-323.

15. Krasinski SD, Cohn JS, Schaefer EJ, et al. (1990) Postprandial plasma retinyl ester response is greater in older subjects compared with younger subjects: evidence for delayed plasma clearance of intestinal lipoproteins. J Clin Invest $\mathbf{8 5}$, 883-892.

16. Silva KDRR, Kelly CNM, Jones AE, et al. (2003) Chylomicron particle size and number, factor VII activation and dietary monounsaturated fatty acids. Atherosclerosis 166, 73-84.

17. Ruf H \& Gould BJ (1998) Size distribution of chylomicrons from human lymph from dynamic light scattering measurements. Eur Biophys J 28, 1-11.

18. Mihas C, Kolovou GD, Mikhailidis DP, et al. (2011) Diagnostic value of postprandial triglyceride testing in healthy subjects: a meta-analysis. Curr Vasc Pharmacol 9, 271-280.

19. Carrel G, Egli L, Tran C, et al. (2011) Contributions of fat and protein to the incretin effect of a mixed meal. Am J Clin Nutr 94, 997-1003.

20. Kameyama N, Maruyama C, Matsui S, et al. (2014) Effects of consumption of main and side dishes with white rice on postprandial glucose, insulin, glucose-dependent insulinotropic polypeptide and glucagon-like peptide- 1 responses in healthy Japanese men. Br J Nutr 111, 1632-1640.

21. Deopurkar R, Ghanim H, Friedman J, et al. (2010) Differential effects of cream, glucose, and orange juice on inflammation, endotoxin, and the expression of toll-like receptor- 4 and suppressor of cytokine signaling-3. Diabetes Care 33, 991-997.

22. Ghanim H, Sia CL, Korzeniewski K, et al. (2011) A resveratrol and polyphenol preparation suppresses oxidative and inflammatory stress response to a high-fat, high-carbohydrate meal. J Clin Endocrinol Metab 96, 1409-1414.

23. Ghanim H, Abuaysheh S, Sia CL, et al. (2009) Increase in plasma endotoxin concentrations and the expression of toll-like receptors and suppressor of cytokine signaling-3 in mononuclear cells after a high-fat, high-carbohydrate 
meal: implications for insulin resistance. Diabetes Care 32 , 2281-2287.

24. Thomsen C, Storm H, Holst JJ, et al. (2003) Differential effects of saturated and monounsaturated fats on postprandial lipemia and glucagon-like peptide 1 responses in patients with type 2 diabetes. Am J Clin Nutr 77, 605-611.

25. Devaraj S, Wang-Polagruto J, Polagruto J, et al. (2008) High-fat, energy-dense, fast-food-style breakfast results in an increase in oxidative stress in metabolic syndrome. Metabolism 57, 867-870.

26. Oikawa S, Mizunuma Y, Iwasaki Y, et al. (2010) Changes of very low-density lipoprotein concentration in hepatic blood from cows with fasting-induced hepatic lipidosis. Can $J$ Vet Res 74, 317-320.

27. Naito HK (1986) Lipoprotein Separations Using the TL-100 Tabletop Ultrafuge. Palo Alto, CA: Beckman Coulter.

28. Kupke IR \& Wörz-Zeugner S (1986) Sequential micro-ultracentrifugation of lipoproteins in 100ul of serum. J Lipid Res 27, 988-995.

29. Fraser R (1970) Size and lipid composition of chylomicrons of different Svedberg units of flotation. J Lipid Res 11, 60-65.

30. Folch J, Lees M \& Sloane Stanley GH (1957) A simple method for the isolation and purification of total lipides from animal tissues. J Biol Chem 226, 497-509.

31. Hamilton JG \& Comai K (1988) Rapid separation of neutral lipids, free fatty acids and polar lipids using prepacked silica Sep-Pak columns. Lipids 23, 1146-1149.

32. Christie WW (1982) A simple procedure for rapid transmethylation of glycerolipids and cholesteryl esters. J Lipid Res 23, 1072-1075.

33. Matthews D, Hosker J, Rudenski A, et al. (1985) Homeostasis model assessment: insulin resistance and B-cell function from fasting plasma glucose and insulin concentrations in man. Diabetologia 28, 412-419.

34. Sakurai T, Trirongjitmoah S, Nishibata Y, et al. (2010) Measurement of lipoprotein particle sizes using dynamic light scattering. Ann Clin Biochem 47, 476-481.

35. Vors C, Pineau G, Gabert L, et al. (2013) Modulating absorption and postprandial handling of dietary fatty acids by structuring fat in the meal: a randomized crossover clinical trial. Am J Clin Nutr 97, 23-36.

36. Mekki N, Christofilis MA, Charbonnier M, et al. (1999) Influence of obesity and body fat distribution on postprandial lipemia and triglyceride-rich lipoproteins in adult women. J Clin Endocrinol Metab 84, 184-191.

37. Sakai N, Uchida Y, Ohashi K, et al. (2003) Measurement of fasting serum apoB-48 levels in normolipidemic and hyperlipidemic subjects by ELISA. J Lipid Res 44, 1256-1262.

38. Iqbal J \& Hussain MM (2009) Intestinal lipid absorption. Am J Physiol Endocrinol Metab 296, E1183-E1194.

39. Duez H, Pavlic M \& Lewis GF (2008) Mechanism of intestinal lipoprotein overproduction in insulin resistant humans. Atheroscler Suppl 9, 33-38.

40. Chen YDI, Swami S, Skowronski R, et al. (1993) Differences in postprandial lipemia between patients with normal glucose tolerance and noninsulin-dependent diabetes mellitus. J Clin Endocrinol Metab 76, 172-177.
41. Quarfordt SH \& Goodman DS (1966) Heterogeneity in the rate of plasma clearance of chylomicrons of different size. Biochim Biophys Acta 116, 382-385.

42. Schafer L, Overvad K, Thorling EB, et al. (1989) Adipose tissue levels of fatty acids and tocopherol in young and old women. Ann Nutr Metab 33, 315-322.

43. Bolton-Smith C, Woodward M \& Tavendale R (1997) Evidence for age-related differences in the fatty acid composition of human adipose tissue, independent of diet. Eur I Clin Nutr 51, 619-624

44. Holman RT, Smythe L \& Johnson S (1979) Effect of sex and age on fatty acid composition of human serum lipids. Am J Clin Nutr 32, 2390-2399.

45. Asciutti-Moura LS, Guilland JC, Fuchs F, et al. (1988) Fatty acid composition of serum lipids and its relation to diet in an elderly institutionalized population. Am J Clin Nutr 48, 980-987.

46. Crowe FL, Skeaff CM, Green TJ, et al. (2008) Serum n-3 long-chain PUFA differ by sex and age in a population-based survey of New Zealand adolescents and adults. Br J Nutr 99 , 168-174.

47. Burdge GC, Powell J \& Calder PC (2006) Lack of effect of meal fatty acid composition on postprandial lipid, glucose and insulin responses in men and women aged 50-65 years consuming their habitual diets. Br J Nutr 96, 489-500.

48. Koutsari C, Zagana A, Tzoras I, et al. (2004) Gender influence on plasma triacylglycerol response to meals with different monounsaturated and saturated fatty acid content. Eur J Clin Nutr 58, 495-502.

49. Hollander D \& Dadufalza VD (1983) Increased intestinal absorption of oleic acid with aging in the rat. Exp Gerontol 18, 287-292

50. Hollander D, Dadufalza VD \& Sletten EG (1984) Does essential fatty acid absorption change with aging? J Lipid Res 25, 129-134.

51. Keelan M, Walker K \& Thomson ABR (1985) Intestinal morphology, marker enzymes and lipid content of brush border membranes from rabbit jejunum and ileum: effect of aging. Mech Ageing Dev 31, 49-68.

52. Ravikumar B, Carey PE, Snaar JEM, et al. (2005) Real-time assessment of postprandial fat storage in liver and skeletal muscle in health and type 2 diabetes. Am J Physiol Endocrinol Metab 288, E789-E797.

53. Chavezjauregui RN, Mattes RD \& Parks EJ (2010) Dynamics of fat absorption and effect of sham feeding on postprandial lipema. Gastroenterology 139, 1538-1548.

54. Matikainen N \& Taskinen MR (2008) Postprandial triglyceriderich lipoproteins in insulin resistance and type 2 diabetes. Future Lipidol 3, 531-543.

55. Davidson NO, Magun AM \& Glickman RM (1991) Enterocyte lipid absorption and secretion. In Handbook of Physiology: The Gastrointestinal System Intestinal Absorption and Secretion, pp. 505-526 [DM Pollock, editor]. Bethesda, MD: American Physiological Society.

56. Laaksonen DE, Lakka TA, Lakka HM, et al. (2002) Serum fatty acid composition predicts development of impaired fasting glycaemia and diabetes in middle-aged men. Diabet Med 19, 456-464. 